\title{
Klotho inhibits H 202 -induced oxidative stress and apoptosis in periodontal ligament stem cells by regulating UCP2 expression
}

\section{Lilei Zhu}

Changsha Stomatological Hospital

Hui Xie

Changsha Stomatological Hospital

Qingqing Liu

Changsha Stomatological Hospital

Fei Ma

Changsha Stomatological Hospital

Hao Wu ( $\nabla$ wuhaocskq@163.com )

Changsha Stomatological Hospital

\section{Research}

Keywords: Klotho, UCP2, Chronic periodontitis

Posted Date: April 28th, 2020

DOl: https://doi.org/10.21203/rs.3.rs-24745/v1

License: (9) This work is licensed under a Creative Commons Attribution 4.0 International License. Read Full License

Version of Record: A version of this preprint was published at Clinical and Experimental Pharmacology and Physiology on July 10th, 2021. See the published version at https://doi.org/10.1111/14401681.13547. 


\section{Abstract}

Background Periodontitis, known as a human chronic inflammatory disease, has affected the life of millions of individuals. Known risk factors such as metabolic disease and oxidative stress have been reported to be closely associated with the initiation or development of periodontitis. However, the etiology of periodontitis remains unclear. Klotho, a single-pass transmembrane protein, has been widely reported to modulate cellular processes in various diseases. However, the role of Klotho in periodontitis is unknown.

Results In this study, we designed and conducted a series of experiments to evaluate the role of Klotho in chronic periodontitis. Our experimental results showed that Klotho was downregulated in the gingival tissues, gingival crevicular fluid (GCF), and periodontal ligament stem cells (PDLSCs) of chronic periodontitis patients. Besides, Klotho upregulated the production of uncoupling protein 2 (UCP2) in $\mathrm{H}_{2} \mathrm{O}_{2^{-}}$ treated PDLSCs. In function, Klotho inhibited $\mathrm{H}_{2} \mathrm{O}_{2}$-induced oxidative stress and cellular apoptosis in PDLSCs. Moreover, the rescue assay suggested that UCP2 knock-down suppressed the effects of Klotho on $\mathrm{H}_{2} \mathrm{O}_{2}$-induced oxidative stress and apoptosis in PDLSCs.

Conclusions In conclusion, we found that Klotho inhibits $\mathrm{H}_{2} \mathrm{O}_{2}$-induced oxidative stress and apoptosis in PDLSCs by regulating UCP2 expression. This novel discovery may provide a potential target for chronic periodontitis treatment.

\section{Background}

Periodontitis is a chronic inflammatory disease characterized with the destruction of the supportive tissues of teeth and periodontal attachment, ultimately resulting in tooth loss, and has affected approximately 740 million individuals worldwide $[1,2]$. Factors such as destructive immune responses and oxidative stress have been reported to be closely associated with the initiation or development of periodontitis [3, 4]. However, the etiology of periodontitis is complicated. Therefore, it is of great urgency to identify potential novel biomarkers for the diagnosis and treatment of this disease.

Given that oxidative stress is characterized by a high level of reactive oxygen species (ROS) [5], the deleterious products induced by ROS activities need evaluation for assessing oxidative stress-related diseases [6]. Moreover, the exacerbation of oxidative stress may play a role in the underlying mechanism for the aggravation of periodontitis [7]. Furthermore, evaluation of antioxidant enzymes including superoxide dismutase (SOD) is required to clarify the complex process of oxidative stress [8].

Klotho, a single-pass transmembrane protein, also known as an anti-aging protein, consists of 1012 amino acids [9-11]. The dysregulation of Klotho has been identified in several diseases and cancers such as chronic kidney disease, left ventricular hypertrophy, and thyroid cancer $[10,12,13]$. Klotho is involved in various biological processes, including the amelioration of fibrosis, alleviation of oxidative stress, and inhibition of cellular apoptosis [14]. Mechanistically, Klotho can regulate several signaling 
pathways such as Wnt/ $\beta$-catenin, NF-kappa B, and transforming growth factor- $\beta 1$ signaling pathways. For example, local cardiac endogenous Klotho improves cardiac fibrosis via TGF- $\beta 1$ and Wnt signaling pathways in humans [15]. Soluble Klotho has been reported to attenuate myocardial hypertrophy induced by FGF23 in mice [16]. Nevertheless, the role of Klotho in periodontitis is unknown.

Uncoupling protein 2 (UCP2), a member of mitochondrial transporters family, has been reported to be involved in metabolic reprogramming $[17,18]$. The ability of UCP2 to regulate glucose is strongly associated with cellular proliferation and apoptosis [19]. Additionally, the UCP2 upregulation may significantly suppress proliferation and migration of vascular smooth muscle cells by inhibiting oxidative stress [20]. However, the biological functions of UCP2 in chronic periodontitis remain unclear.

In the current study, we aimed to investigate the role of Klotho and UCP2 in chronic periodontitis and discovered that Klotho inhibits $\mathrm{H}_{2} \mathrm{O}_{2}$-induced oxidative stress and apoptosis in periodontal ligament stem cells (PDLSCs) by regulating UCP2 expression. This novel discovery may provide a potential target for chronic periodontitis treatment.

\section{Materials And Methods}

\section{Sample collection}

The current study included 12 patients with periodontitis (patient group, 7 males and 5 females) and 10 patients without periodontitis but with orthodontic demands (control group, 5 males, 5 females). Patients with more than $3 \mathrm{~mm}$ periodontal pocket depth were diagnosed as periodontal disease. Patients who had received any treatment or combined with other diseases were excluded from this study. Gingival crevicular fluid (GCF) samples and gingival tissues were obtained from patients $(n=22)$ of Changsha Stomatological Hospital. GCF and gingival tissue were isolated and collected as described before [21, 22]. Consent forms were obtained before surgery. This study has been approved by the Ethics Committee of Changsha Stomatological Hospital.

\section{Cell culture and transfection}

PDLSCs were separated from gingival tissues of patients with orthodontic demands as described before $[23,24]$. Cells were cultivated in $\alpha$-minimal essential medium ( $\alpha$-MEM; Gibco BRL, USA) and supplemented with 10\% fetal bovine serum (Gibco BRL), 100 units/ml penicillin (Invitrogen Life Technology, USA), 100 $\mathrm{mg} / \mathrm{ml}$ streptomycin (Invitrogen), and $0.292 \mathrm{mg} / \mathrm{ml} \mathrm{l-glutamine} \mathrm{(Invitrogen)} \mathrm{at} 37^{\circ} \mathrm{C}$ with $5 \% \mathrm{CO}_{2}$. To induce oxidative stress, PDLSCs were treated with $\mathrm{H}_{2} \mathrm{O}_{2}(100 \mu \mathrm{M})$ for $24 \mathrm{~h}$ before each experiment. To evaluate the effect of Klotho on PDLSCs, cells were treated with Klotho (final concentration: $10 \mathrm{ng} / \mathrm{mL}$ for $48 \mathrm{~h}$, and cells treated with the same concentration of DMSO served as controls. Short-interfering RNA (siRNA)-targeted UCP2 (si-UCP2) was purchased from GenePharma (Shanghai, China) and transfected into cells using lipofectamine 2000 (Invitrogen).

\section{ELISA}


The level of Klotho in GCF samples (or controls) and PDLSCs, as well as the levels of ROS, malondialdehyde (MDA), SOD, and glutathione peroxidase (GSH-PX) in PDLSCs, were assessed using an ELISA kit (R\&D Systems, Inc.) following the manufacturer's instructions.

\section{Western blot assay}

Protein samples from cultured PDLSCs or gingival tissues were acquired using the Radio Immunoprecipitation Assay (RIPA) lysis buffer (Beyotime, Beijing, China) and isolated on 10\% SDS-PAGE. After moving onto polyvinylidene fluoride (PVDF membranes, 5\% non-fat milk was added. Later, samples were treated with primary antibodies for Klotho (ab181373, Abcam, Cambridge, USA), UCP2 (ab97931), Bcl-2 (ab32124), Bax (ab32503), cleaved caspase-3 (ab2302), and GAPDH (ab8245, Abcam) overnight. Subsequently, secondary antibodies were added to the culture in darkness for $1 \mathrm{~h}$. Finally, proteins were evaluated using a chemiluminescence detection system.

\section{Terminal deoxynucleotidyl transferase dUTP nick end labeling (TUNEL) assay}

According to the protocol of TUNEL assay, apoptosis of PDLSCs was detected using the In Situ Cell Death Detection kit (Roche). Apoptosis was evaluated by counting the positive cells as well as total number of cells in five random fields. Cell nuclei were positive if they were labelled with FITC (green), and DAPI staining indicated the cell nuclei under a florescence microscope. Positively stained cells were counted using image-Pro Plus 6.0 software.

\section{Statistical analysis}

All experiments were performed at least three times $(N \geq 3)$. To analyze the statistical difference, the $P$ values between any two groups were calculated by one-way analysis of variance (ANOVA) using Graphpad 6.0 statistical software (GraphPad Software Inc., San Diego, CA, USA). The results of multiple experiments are presented as mean \pm standard deviation. The result was considered statistically significant when $\mathrm{P}<0.05$.

\section{Results}

\section{Klotho expression in GCF and gingival tissue of patients with chronic periodontitis}

Although Klotho has been widely reported to alleviate cardiovascular disease and chronic kidney disease, its expression levels and biological function in chronic periodontitis remained uncharacterized. In the current study, we discovered that Klotho levels in GCF of periodontitis patients were significantly lower than those in the healthy counterparts (Fig. 1A). Additionally, the protein level was also downregulated in the gingival tissues of patients with chronic periodontitis compared with the level in controls (Fig.1B). Taken together, Klotho displayed a relatively low level in GCF and gingival tissue of patients with chronic periodontitis. 


\section{UCP2 expression in the gingival tissue of patients with chronic periodontitis}

We then evaluated the levels of UCP2 in gingival tissues. As shown in Fig. 2, the protein level of UCP2 in the gingival tissue of patients with chronic periodontitis was much lower than that in the gingival tissue of the controls.

\section{Klotho upregulates UCP2 in H2O2-treated PDLSCs}

Based on the results of ELISA, Klotho concentration in PDLSCs was markedly reduced by $\mathrm{H}_{2} \mathrm{O}_{2}$ treatment, and the result was reversed by the overexpression of Klotho (Fig. 3A). Likewise, $\mathrm{H}_{2} \mathrm{O}_{2}$ treatment caused an evident reduction in Klotho and UCP2 protein levels, and this reduction was compensated by inducing Klotho overexpression (Fig. 3B-D). Collectively, Klotho upregulated UCP2 level in $\mathrm{H}_{2} \mathrm{O}_{2}$-treated PDLSCs.

\section{Klotho inhibits H2O2-induced oxidative stress in PDLSCs}

$\mathrm{H}_{2} \mathrm{O}_{2}$ treatment induced an apparent rise in the ROS levels in PDLSCs, and this rise was repressed by Klotho overexpression (Fig. 4A). Further, MDA levels displayed similar alterations (Fig. 4B). In contrast, SOD and GSH-PX levels decreased with $\mathrm{H}_{2} \mathrm{O}_{2}$ treatment in PDLSCs, and the levels were restored by pcDNA3.1/Klotho transfection (Fig. 4C-D). In conclusion, Klotho inhibited $\mathrm{H}_{2} \mathrm{O}_{2}$-induced 4Aoxidative stress in PDLSCs by upregulating ROS and MDA, but downregulating 4ASOD and GSH-PX.

\section{Klotho inhibits H2O2-induced apoptosis in PDLSCs}

We further explored the effect of Klotho on cellular apoptosis in chronic periodontitis. Based on the results of TUNEL assay, the enhancement of TUNEL-positive cells induced by $\mathrm{H}_{2} \mathrm{O}_{2}$ treatment was repressed by the addition of Klotho (Fig. 5A). Furthermore, $\mathrm{H}_{2} \mathrm{O}_{2}$-mediated downregulation of $\mathrm{Bcl}-2$, upregulation of Bax, and cleavage of caspase 3 were neutralized by Klotho treatment (Fig. 5B). In summary, Klotho inhibited $\mathrm{H}_{2} \mathrm{O}_{2}$-induced apoptosis in PDLSCs.

\section{si-UCP2 suppresses the effects of Klotho on H2O2-induced oxidative stress and apoptosis in PDLSCs}

To further explore the effects of Klotho and UCP2 on cellular apoptosis and oxidative stress, rescue assays were performed. At the beginning, a satisfactory knockdown efficacy of si-UCP2 was validated by Western blot assay (Fig. 6A). Further, the inhibitory effect of Klotho on ROS and MDA levels was counteracted by silencing UCP2 in PCLSCs treated with $\mathrm{H}_{2} \mathrm{O}_{2}$ (Fig. 6B-C). In contrast, the Klotho-induced rise of SOD and GSH-PX levels was offset by UCP2 inhibition (Fig. 6D-E). Moreover, TUNEL assay using $\mathrm{H}_{2} \mathrm{O}_{2}$-induced PCLSCs revealed that the knock-down of UCP2 restored the promotive impact of Klotho treatment on cellular apoptosis (Fig. 6F-G). In addition, the augmentation of Bcl-2, decline of Bax, and cleavage of caspase 3 resulting from Klotho treatment were countervailed by UCP2 inhibition (Fig. $6 \mathrm{H}-\mathrm{K}$ ). Briefly, si-UCP2 suppressed the effects of Klotho on $\mathrm{H}_{2} \mathrm{O}_{2}$-induced oxidative stress and apoptosis in PDLSCs. 


\section{Discussion}

Periodontitis, a human chronic inflammatory disease, has affected the life of millions of people [25]. Although antibiotic therapies are effective in reducing periodontal destruction, it is still extremely difficult to cure chronic periodontitis given the complex pathogenesis and bacterial resistance [26]. In addition, only few periodontitis patients undergo surgical intervention. PDLSCs, derived from the periodontal ligament, have tissue specificity and impaired differentiation ability and are closely associated with periodontitis [27]. In the present study, we isolated PDLSCs from gingival tissues to evaluate the role of Klotho and UCP2 in chronic periodontitis.

In this study, we presented that Klotho expression was significantly decreased in GCF and gingival tissues of patients with chronic periodontitis, implying that Klotho may alleviate chronic periodontitis. Similarly, UCP2 expression was also low in the gingival tissues of patients. Based on previous studies, Klotho inhibits oxidative stress in renal diseases, heart diseases, and lung diseases [28-30]. In our study, we treated PDLSCs with $\mathrm{H}_{2} \mathrm{O}_{2}(100 \mu \mathrm{M})$ to induce oxidative stress. Consistent with previous studies, $\mathrm{H}_{2} \mathrm{O}_{2}$ treatment lowered Klotho and UCP2 levels. However, treatment with Klotho reversed the decline in UCP2 protein level. Besides, the biomarkers of oxidative stress including ROS, MDA, SOD, and GSH-PX were reduced by $\mathrm{H}_{2} \mathrm{O}_{2}$ treatment, and then abrogated by Klotho treatment.

The typical symptom of periodontitis is the destruction or loss of the supportive tissues of teeth [31]. Moreover, apoptosis of PDLSCs is closely correlated with the destruction or loss of the supportive tissues of teeth [32]. Thus, we evaluated the function of Klotho protein on cellular apoptosis under oxidative stress. We observed that Klotho reversed the promotive effect of $\mathrm{H}_{2} \mathrm{O}_{2}$ on cellular apoptosis by decreasing Bax, cleaving caspase -3 , and increasing Bcl-2 protein levels in PDLSCs.

UCP2 has also been reported to regulate oxidative stress in other diseases. For instance, UCP2 deficiency promotes oxidative stress in cardiovascular diseases [33]. Likewise, in our study, the knock-down of UCP2 suppressed the effects of Klotho on $\mathrm{H}_{2} \mathrm{O}_{2}$-induced oxidative stress and apoptosis in PDLSCs.

\section{Conclusion}

In conclusion, for the first time, we have studied the biological role of Klotho in chronic periodontitis and confirm that $\mathrm{Klotho}$ inhibits $\mathrm{H}_{2} \mathrm{O}_{2}$-induced oxidative stress and apoptosis in PDLSCs by regulating the expression of UCP2. This novel discovery may provide a potential target for the treatment of chronic periodontitis. However, the molecular mechanisms of Klotho in chronic periodontitis remain to be evaluated in future studies.

\section{Abbreviations}

GCF: gingival crevicular fluid; PDLSCs: periodontal ligament stem cells; UCP2: uncoupling protein 2; ROS: reactive oxygen species; SOD: superoxide dismutase; $\alpha$-MEM: $\alpha$-minimal essential medium; si-RNA: Short- 
interfering RNA; si-UCP2: Short-interfering UCP2; MDA: malondialdehyde; GSH-PX: glutathione peroxidase; RIPA: Radio Immunoprecipitation Assay; PVDF: polyvinylidene fluoride; TUNEL: transferase dUTP nick end labeling; ANOVA: analysis of variance

\section{Declarations}

\section{Ethics approval and consent to participate}

Consent forms were obtained before surgery. This study has been approved by the Ethics Committee of Changsha Stomatological Hospital.

\section{Consent for publication}

All participants gave their signed informed consent before entering in the study.

\section{Availability of data and material}

The data used to support the findings of this study are available from the corresponding author upon request.

\section{Competing interests}

The authors declare that they have no competing interests.

\section{Funding}

This research was financially supported by the Basic research project of Changsha science and technology bureau [kq1901013].

\section{Authors' contributions}

LLZ: Methodology, Investigation, Datas Curation, Writing - Original Draft, Writing - Review \& Editing, Visualisation. HX: Formal Analysis, Conceptualization, Validation. QQL: Conceptualization, Investigation, Resources. FM: Investigation, Resources, Dats Curation. HW: Conceptulization, Methodology, Validation, Resources, Investigation, Writing - Review \& Editing, Project Administration, Supervision. All authors read and approved the final manuscript.

\section{Acknowledgements}

We appreciate the support of Changsha Stomatological Hospital. 


\section{References}

1. Slots J. Periodontitis: facts, fallacies and the future. Periodontol 2000. 2017;75:7-23.

2. Papapanou PN, Susin C. Periodontitis epidemiology: is periodontitis under-recognized, overdiagnosed, or both? Periodontol 2000. 2017; 75:45-51.

3. Pihlstrom BL, Michalowicz BS, Johnson NW. Periodontal diseases. Lancet. 2005;366:1809-20.

4. Stadler AF, Angst PD, Arce RM, Gomes SC, Oppermann RV, Susin C. Gingival crevicular fluid levels of cytokines/chemokines in chronic periodontitis: a meta-analysis. J Clin Periodontol. 2016;43:727-45.

5. Sies H. Oxidative stress: a concept in redox biology and medicine. Redox Biol. 2015;4:180-3.

6. Newsholme P, Cruzat VF, Keane KN, Carlessi R, de Bittencourt PI. Jr. Molecular mechanisms of ROS production and oxidative stress in diabetes. Biochem J. 2016;473:4527-50.

7. Boia S, Boariu M, Baderca F, Rusu D, Muntean D, Horhat F, Boia ER, Borza C, Anghel A, Stratul SI. Clinical, microbiological and oxidative stress evaluation of periodontitis patients treated with two regimens of systemic antibiotics, adjunctive to non-surgical therapy: A placebo-controlled randomized clinical trial. Exp Ther Med. 2019;18:5001-15.

8. Poprac P, Jomova K, Simunkova M, Kollar V, Rhodes CJ, Valko M. Targeting Free Radicals in Oxidative Stress-Related Human Diseases. Trends Pharmacol Sci. 2017;38:592-607.

9. Torbus-Paluszczak M, Bartman W, Adamczyk-Sowa M. Klotho protein in neurodegenerative disorders. Neurol Sci. 2018;39(10):1677-82.

10. Zou D, Wu W, He Y, Ma S, Gao J. The role of klotho in chronic kidney disease. BMC Nephrol. 2018;19:285.

11. Boksha IS, Prokhorova TA, Savushkina OK, Tereshkina EB. Klotho Protein: Its Role in Aging and Central Nervous System Pathology. Biochemistry. 2017;82:990-1005.

12. Yang K, Wang C, Nie L, Zhao X, Gu J, Guan X, Wang S, Xiao T, Xu X, He T, Xia X, Wang J, Zhao J. Klotho Protects Against Indoxyl Sulphate-Induced Myocardial Hypertrophy. J Am Soc Nephrol. 2015;26:2434-46.

13. Kang YE, Kim JT, Lim MA, Oh C, Liu L, Jung SN, Won HR, Lee K, Chang JW, Yi HS, Kim HJ, Ku BJ, Shong M, Koo BS. Association between Circulating Fibroblast Growth Factor 21 and Aggressiveness in Thyroid Cancer. Cancers (Basel). 2019;11.

14. Mencke R, Olauson H, Hillebrands JL. Effects of Klotho on fibrosis and cancer: A renal focus on mechanisms and therapeutic strategies. Adv Drug Deliv Rev. 2017;121:85-100.

15. Liu Q, Zhu LJ, Waaga-Gasser AM, Ding Y, Cao M, Jadhav SJ, Kirollos S, Shekar PS, Padera RF, Chang YC, Xu X, Zeisberg EM, Charytan DM, Hsiao LL. The axis of local cardiac endogenous Klotho-TGFbeta1-Wnt signaling mediates cardiac fibrosis in human. J Mol Cell Cardiol. 2019;136:113-24.

16. Lu X, Hu MC. Klotho/FGF23 Axis in Chronic Kidney Disease and Cardiovascular Disease. Kidney Dis (Basel). 2017;3(1):15-23. 
17. Donadelli M, Dando I, Fiorini C, Palmieri M. UCP2, a mitochondrial protein regulated at multiple levels. Cell Mol Life Sci. 2014;71:1171-90.

18. Broche B, Ben Fradj S, Aguilar E, Sancerni T, Benard M, Makaci F, Berthault C, Scharfmann R, AlvesGuerra MC, Duvillie B. Mitochondrial Protein UCP2 Controls Pancreas Development. Diabetes. 2018;67:78-84.

19. Zhou Y, Cai T, Xu J, Jiang L, Wu J, Sun Q, Zen K, Yang J. UCP2 attenuates apoptosis of tubular epithelial cells in renal ischemia-reperfusion injury. Am J Physiol Renal Physiol. 2017;313:F926-37.

20. Zhou Y, Zhang MJ, Li BH, Chen L, Pi Y, Yin YW, Long CY, Wang X, Sun MJ, Chen X, Gao CY, Li JC, Zhang LL. PPARgamma Inhibits VSMC Proliferation and Migration via Attenuating Oxidative Stress through Upregulating UCP2. PLoS One. 2016;11:e0154720.

21. Offenbacher S, Odle BM, Van Dyke TE. The use of crevicular fluid prostaglandin E2 levels as a predictor of periodontal attachment loss. J Periodontal Res. 1986;21:101-12.

22. Uematsu S, Mogi M, Deguchi T. Interleukin (IL)-1 beta, IL-6, tumor necrosis factor-alpha, epidermal growth factor, and beta 2-microglobulin levels are elevated in gingival crevicular fluid during human orthodontic tooth movement. J Dent Res. 1996;75:562-7.

23. Liu Y, Liu W, Hu C, Xue Z, Wang G, Ding B, Luo H, Tang L, Kong X, Chen X, Liu N, Ding Y, Jin Y. MiR-17 modulates osteogenic differentiation through a coherent feed-forward loop in mesenchymal stem cells isolated from periodontal ligaments of patients with periodontitis. Stem Cells. 2011;29:180416.

24. Zhang J, An Y, Gao LN, Zhang YJ, Jin Y, Chen FM. The effect of aging on the pluripotential capacity and regenerative potential of human periodontal ligament stem cells. Biomaterials. 2012;33:697486.

25. Manresa C, Sanz-Miralles EC, Twigg J, Bravo M. Supportive periodontal therapy (SPT) for maintaining the dentition in adults treated for periodontitis. Cochrane Database Syst Rev. 2018;1:Cd009376.

26. Zhu L, Yao Y, Liu J, Wang J, Xie H. Expression of beta-catenin and MMP-8 in gingival crevicular fluid and gingival tissue indicates the disease severity of patients with chronic periodontitis. Exp Ther Med. 2019;18:2131-9.

27. Hu L, Liu Y, Wang S. Stem cell-based tooth and periodontal regeneration. Oral Dis. 2018;24:696-705.

28. Qian Y, Guo X, Che L, Guan X, Wu B, Lu R, Zhu M, Pang H, Yan Y, Ni Z, Gu L. Klotho Reduces Necroptosis by Targeting Oxidative Stress Involved in Renal Ischemic-Reperfusion Injury. Cell Physiol Biochem. 2018;45:2268-82.

29. Yao Y, Wang Y, Zhang Y, Liu C. Klotho ameliorates oxidized low density lipoprotein (ox-LDL)-induced oxidative stress via regulating LOX-1 and PI3K/Akt/eNOS pathways. Lipids Health Dis. 2017;16:77.

30. Hsia CCW, Ravikumar P, Ye J. Acute lung injury complicating acute kidney injury: A model of endogenous alphaKlotho deficiency and distant organ dysfunction. Bone. 2017;100:100-9.

31. Smith MM, Knight ET, Al-Harthi L, Leichter JW. Chronic periodontitis and implant dentistry. Periodontol 2000. 2017; 74:63-73. 
32. Han J, Menicanin D, Gronthos S, Bartold PM. Stem cells, tissue engineering and periodontal regeneration. Aust Dent J. 2014;59:117-30.

33. Tian XY, Ma S, Tse G, Wong WT, Huang Y. Uncoupling Protein 2 in Cardiovascular Health and Disease. Front Physiol. 2018;9:1060.

\section{Figures}
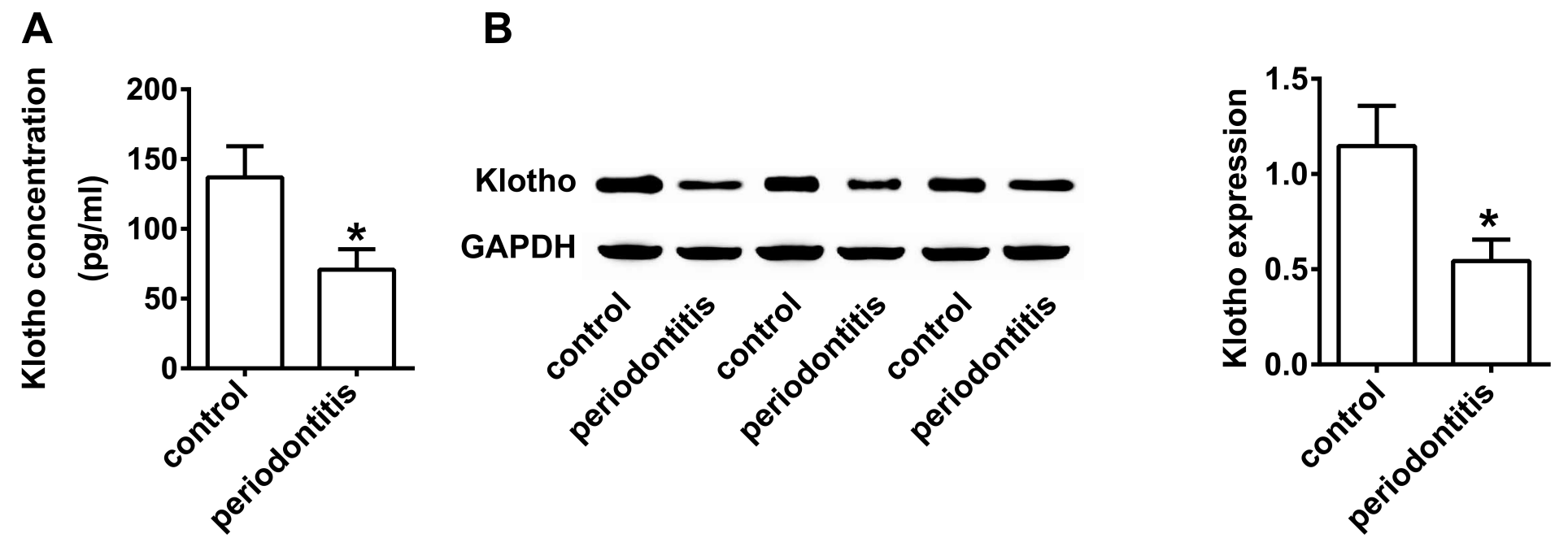

Figure 1

The expression Klotho in GCF and gingival tissue of patients with chronic periodontitis (A) ELISA assay is used to measure Klotho concentration in GCF. (B) Western blot assay is performed to detect Klotho levels in gingival tissues. ${ }^{*}<<0.05$ vs control
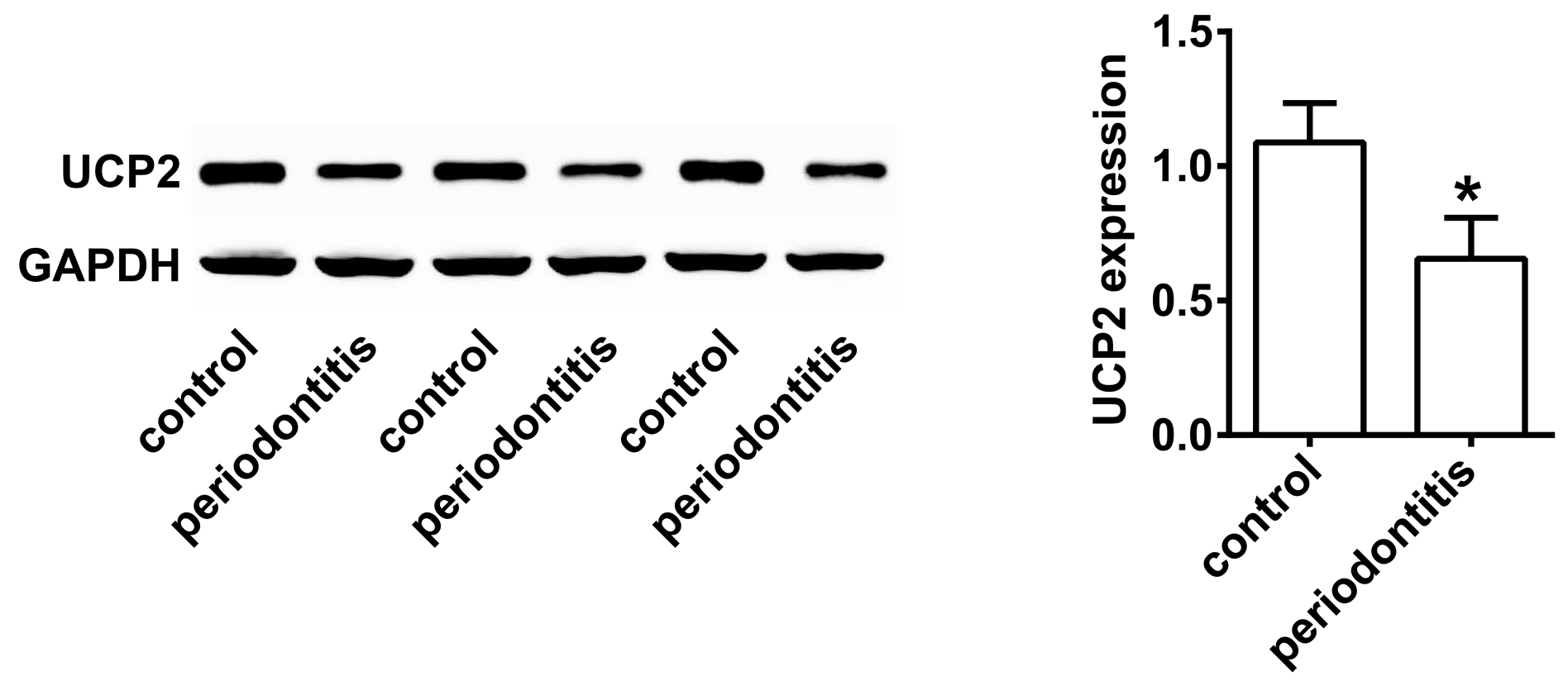
Figure 2

The expression UCP2 in gingival tissue of patients with chronic periodontitis UCP2 protein level is tested by Western blot assay. ${ }^{*} \mathrm{P}<0.05$ vs control

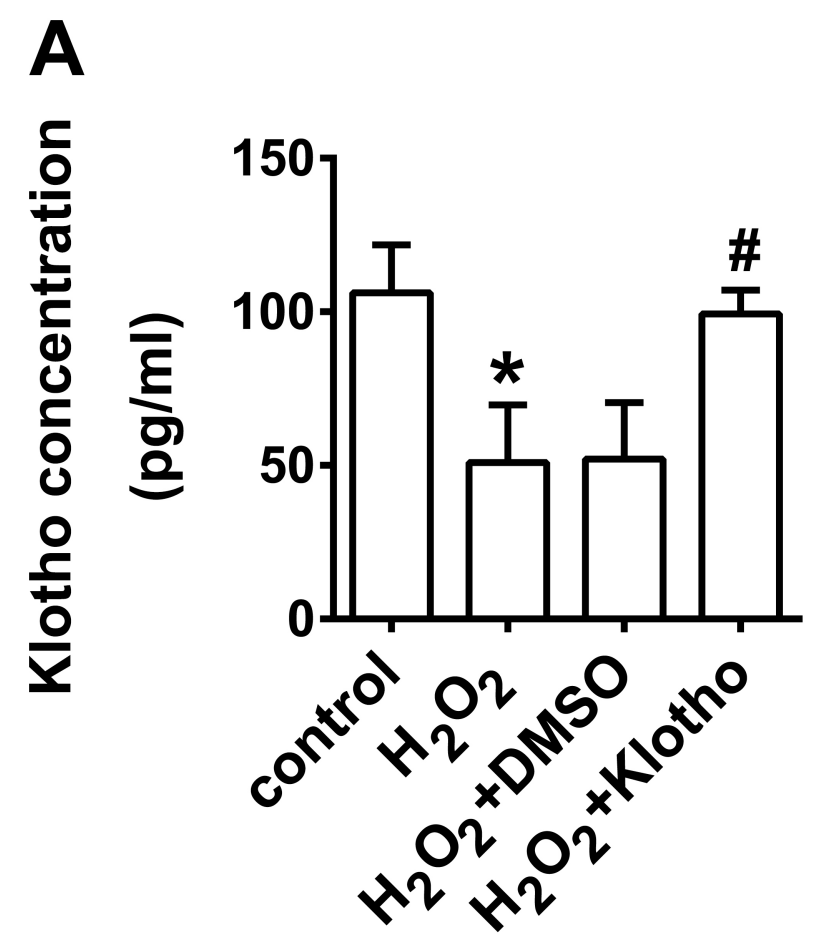

B
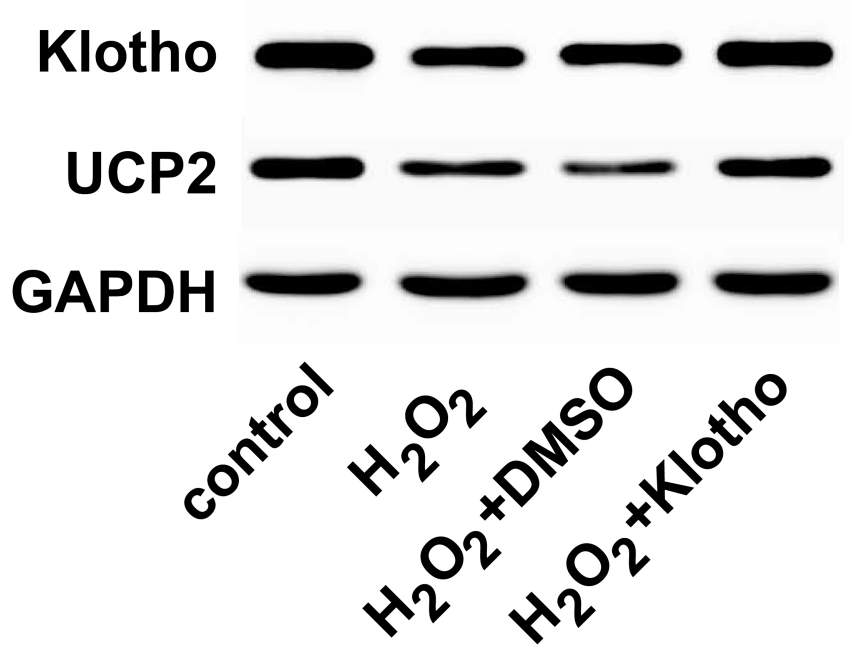

C

D
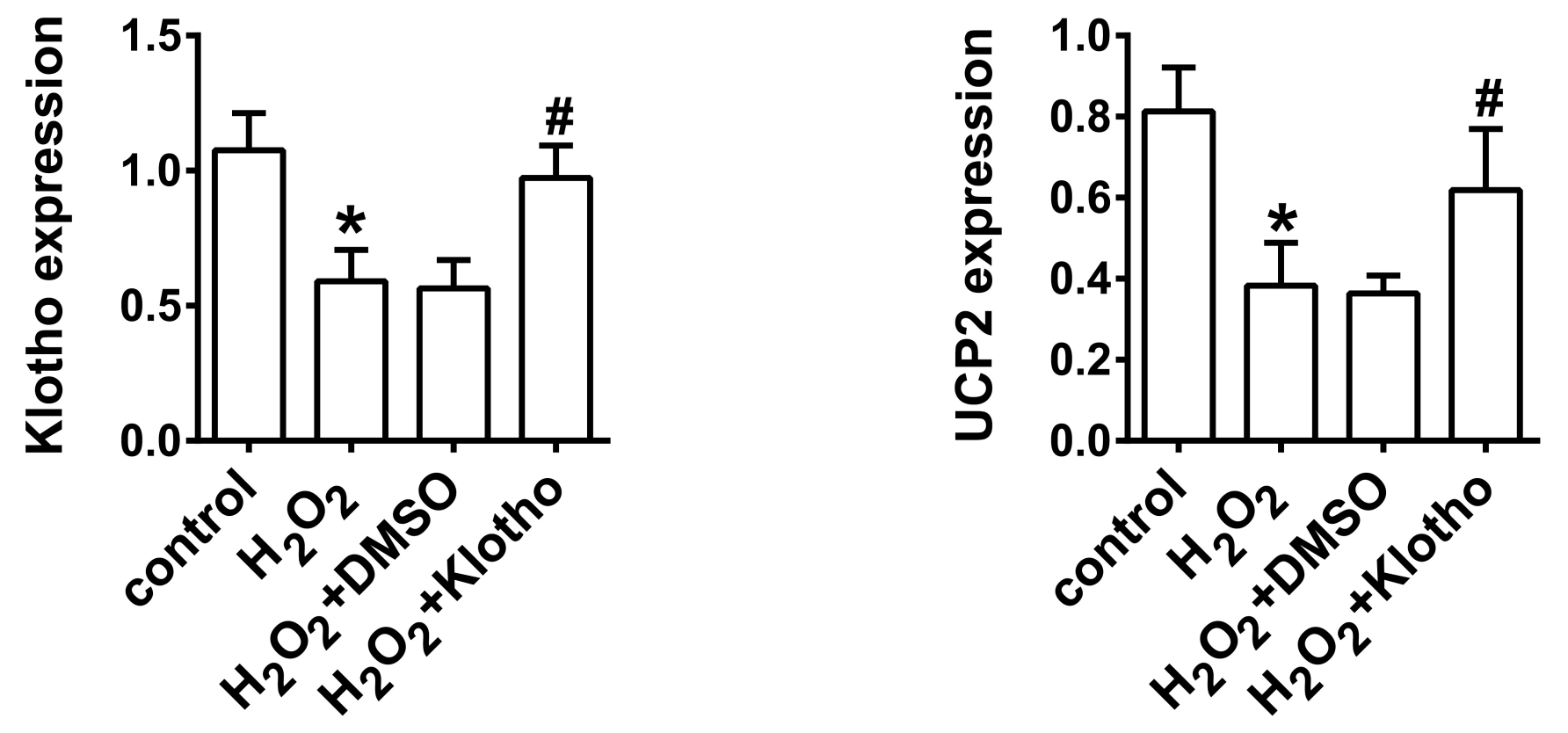

Figure 3 
Klotho up-regulates UCP2 in H2O2-treated PDLSCs (A) Klotho concentration in PDLSCs is measured by ELISA. (B-D) The protein levels of UCP2 and Klotho in PDLSCs are tested by Western blot assay. ${ }^{*} \mathrm{P}<0.05$ vs control; $\# \mathrm{P}<0.05$ vs H2O2+DMSO

A
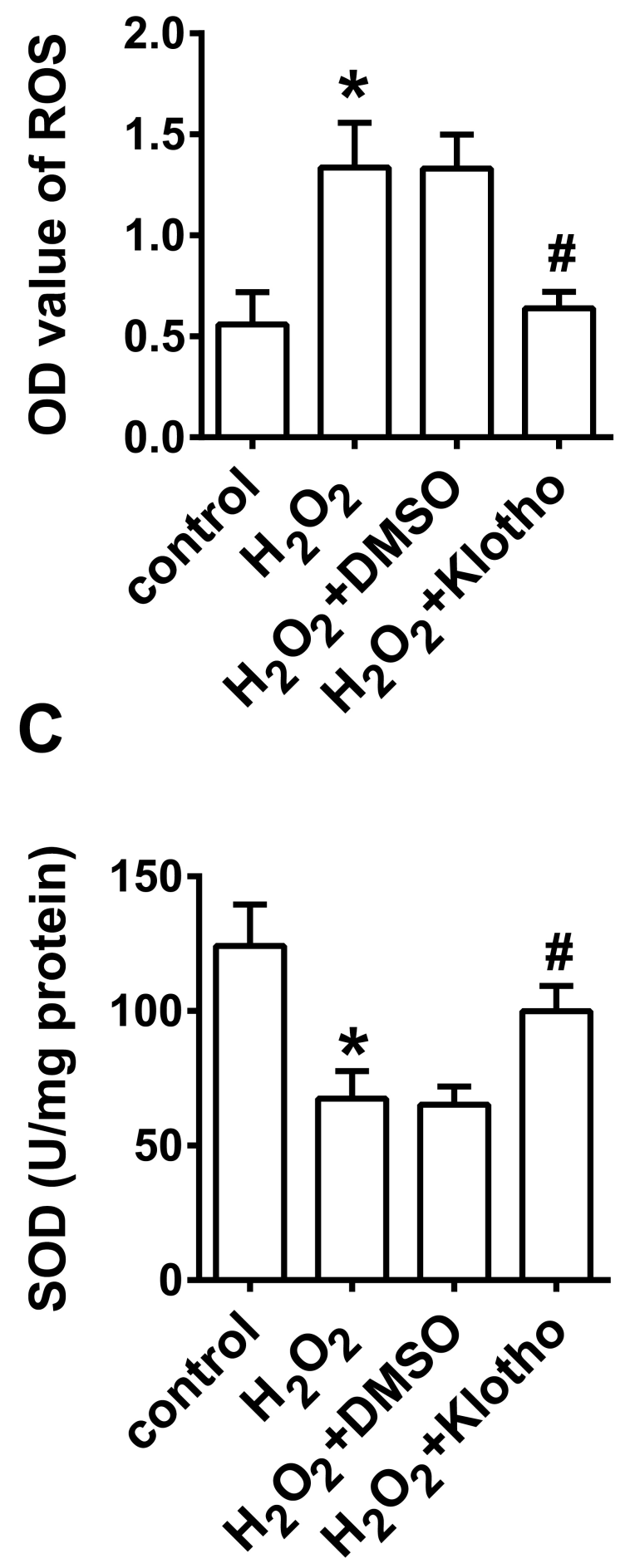

B
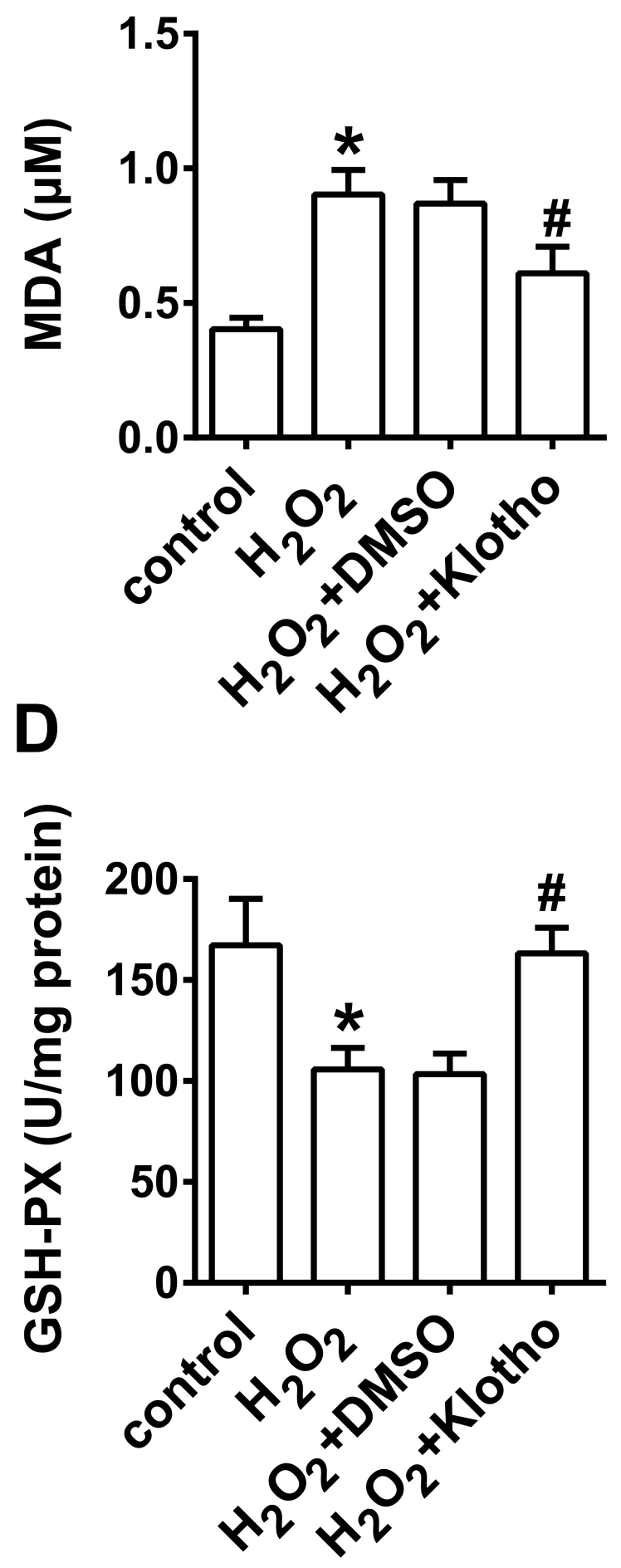

Figure 4

Klotho inhibits H2O2-induced oxidative stress in PDLSCs (A-D) ROS, MDA, SOD, and GSH-PX levels in PDLSCs after treatment with $\mathrm{H} 2 \mathrm{O} 2$ as determined by ELISA. ${ }^{*} \mathrm{P}<0.05$ vs control; \#P $<0.05$ vs 
A
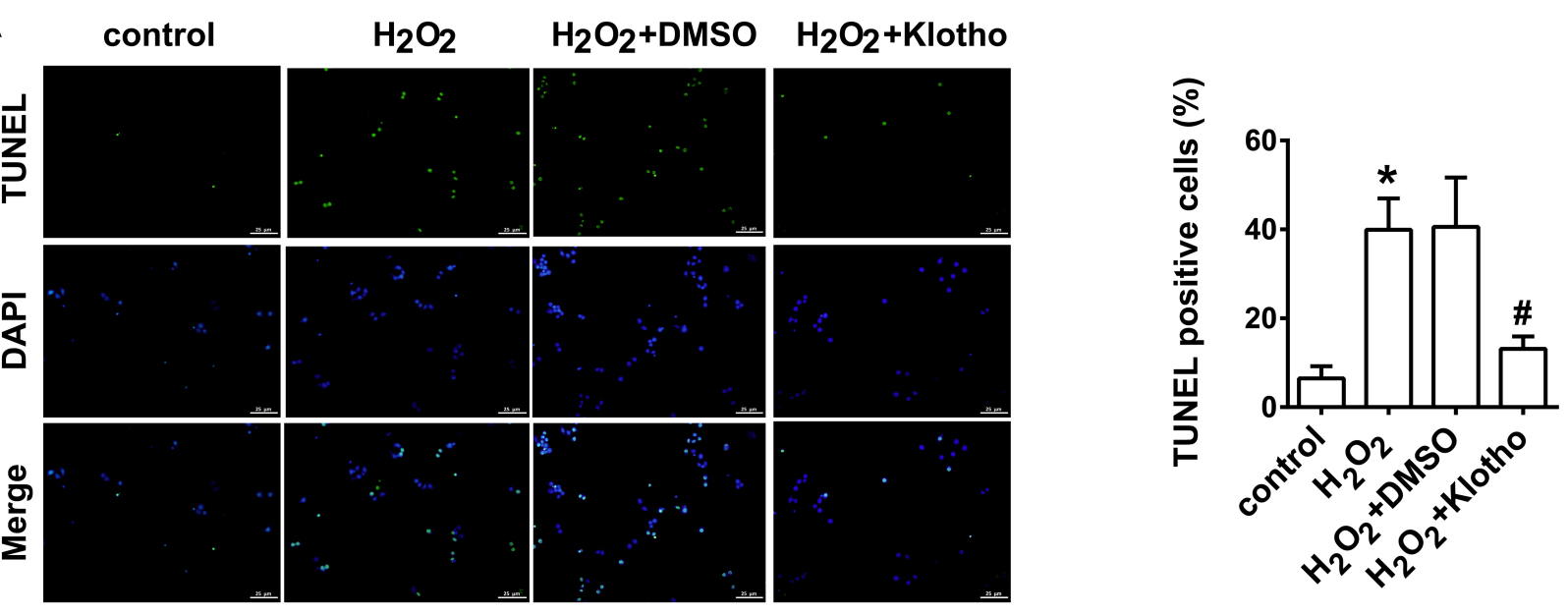
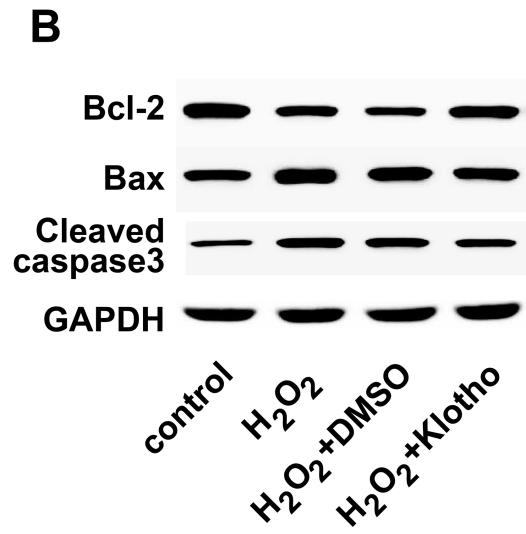
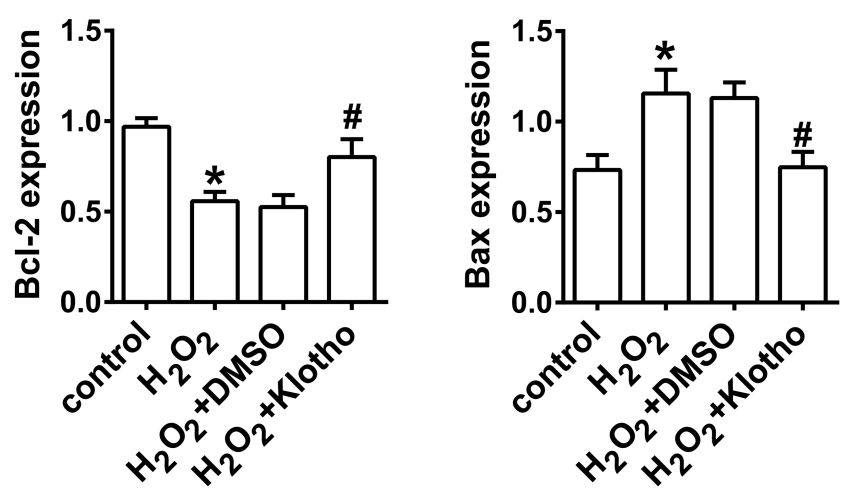

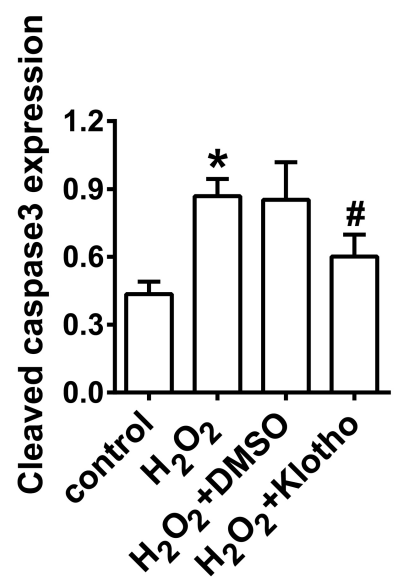

Figure 5

Klotho inhibits H2O2-induced apoptosis in PDLSCs (A) TUNEL assay is used to evaluate cellular apoptosis of PDLSCs. (B) The protein levels of Bcl-2, Bax, and cleaved caspase3 are measured by Western blot assay. ${ }^{*} \mathrm{P}<0.05$ vs control; $\# \mathrm{P}<0.05$ vs H2O2+DMSO 


\section{A}

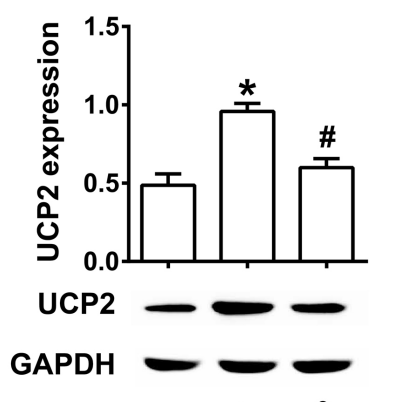

B

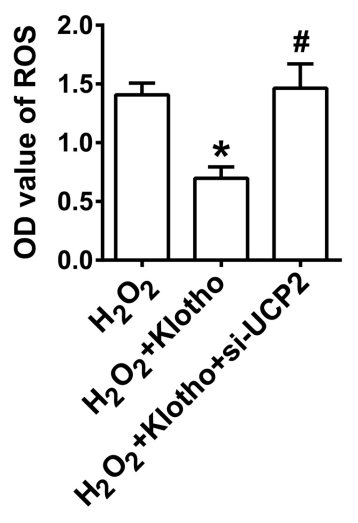

C

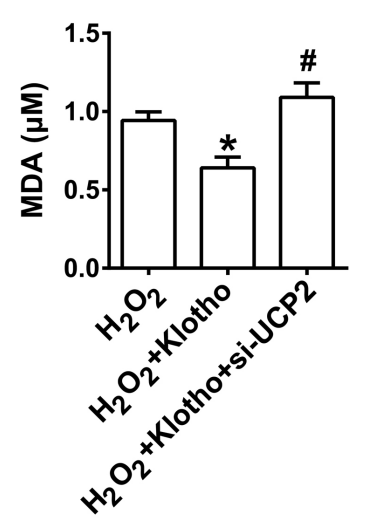

D

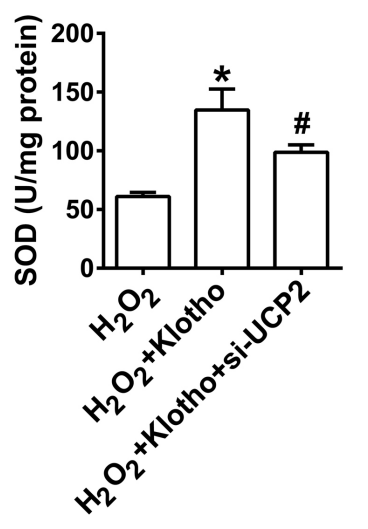

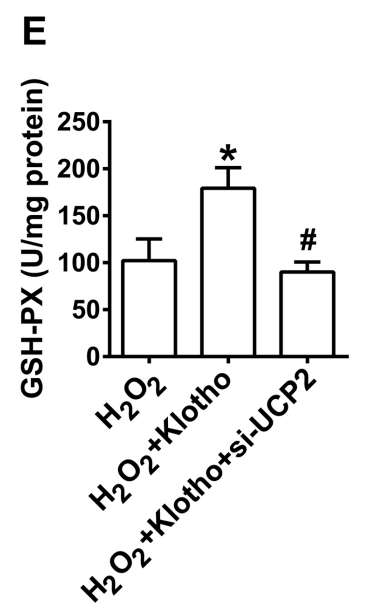

F $\quad \mathrm{H}_{2} \mathrm{O}_{2} \quad \mathrm{H}_{2} \mathrm{O}_{2}+$ Klotho $\mathrm{H}_{2} \mathrm{O}_{2}$ +Klotho+si-UCP2 $\mathrm{G}$
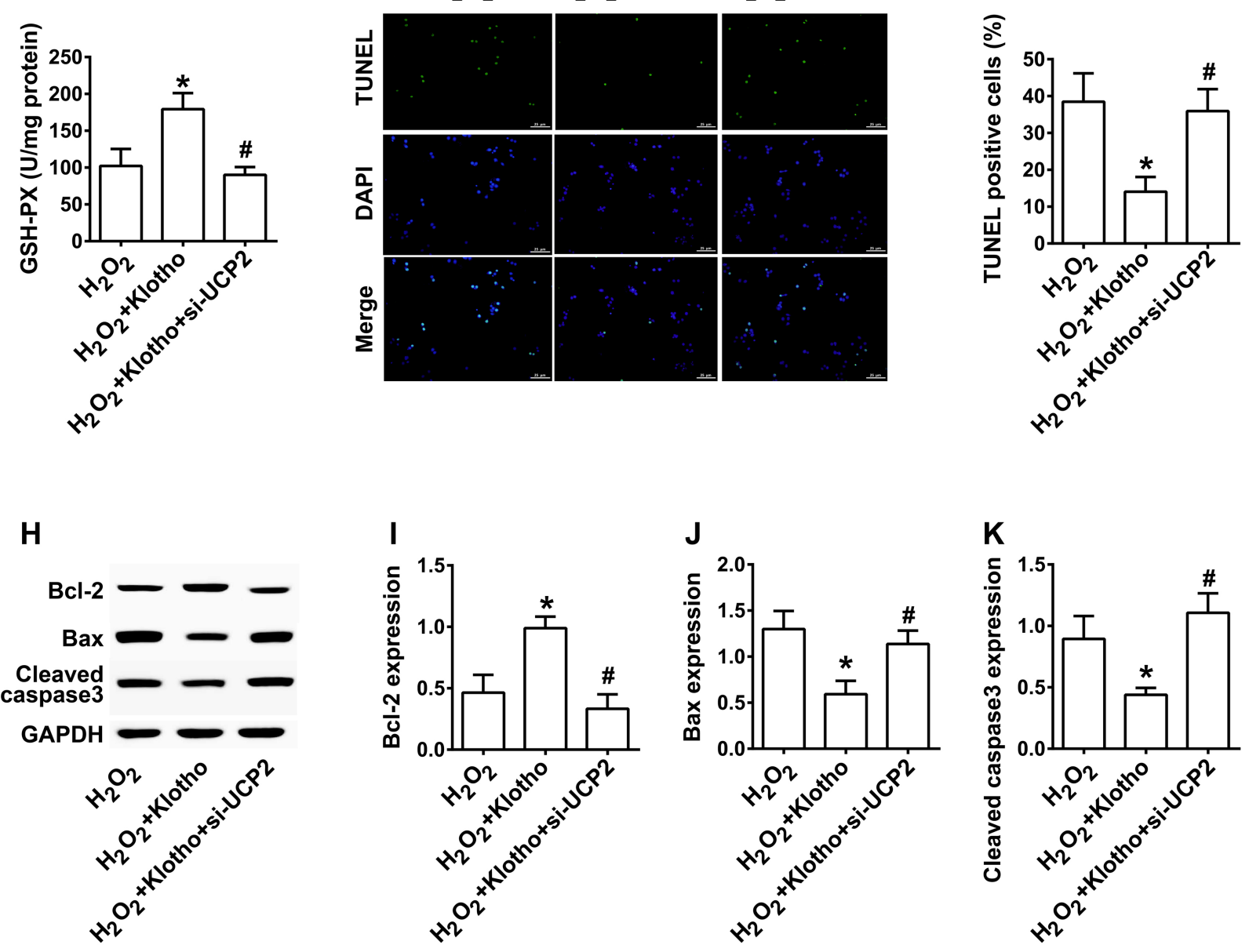

Figure 6

Small interfering RNA of UCP2 suppresses the effects of Klotho on H2O2-induced oxidative stress and apoptosis in PDLSCs (A) The detection of UCP2 protein level in PDLSCs is performed by Western blot assay. (B-E) The measurement of ROS, MDA, SOD, and GSH-PX in PDLSCs is conducted by ELISA. (F-G) Cellular apoptosis is analyzed by TUNEL assay. (H-K) Western blot assay is used to assess the protein levels of $\mathrm{Bcl}-2$, Bax, and cleaved caspase3. ${ }^{*} \mathrm{P}<0.05$ vs H2O2; $\mathrm{AP}<0.05$ vs H2O2+Klotho 